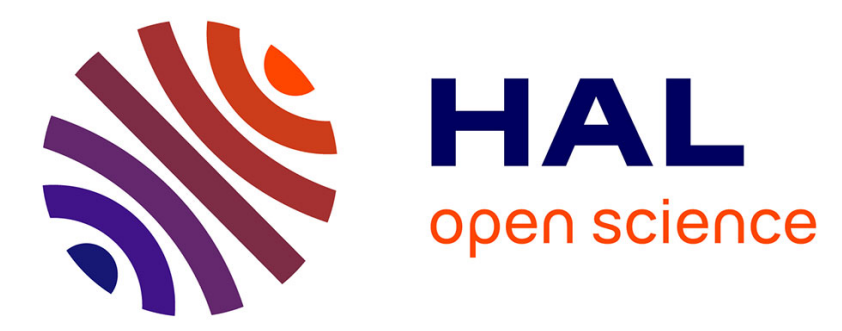

\title{
Détermination des caractéristiques thermophysiques d'une argile prélevée par carottage et d'un mortier en régimes transitoire et asymptotique. Méthode du film chaud cylindrique
}

G. Bastian

\section{- To cite this version:}

G. Bastian. Détermination des caractéristiques thermophysiques d'une argile prélevée par carottage et d'un mortier en régimes transitoire et asymptotique. Méthode du film chaud cylindrique. Revue de Physique Appliquée, 1989, 24 (11), pp.1057-1068. 10.1051/rphysap:0198900240110105700 . jpa00246142

HAL Id: jpa-00246142

https://hal.science/jpa-00246142

Submitted on 1 Jan 1989

HAL is a multi-disciplinary open access archive for the deposit and dissemination of scientific research documents, whether they are published or not. The documents may come from teaching and research institutions in France or abroad, or from public or private research centers.
L'archive ouverte pluridisciplinaire HAL, est destinée au dépôt et à la diffusion de documents scientifiques de niveau recherche, publiés ou non, émanant des établissements d'enseignement et de recherche français ou étrangers, des laboratoires publics ou privés. 


\title{
Détermination des caractéristiques thermophysiques d'une argile prélevée par carottage et d'un mortier en régimes transitoire et asymptotique. Méthode du film chaud cylindrique
}

\author{
G. Bastian (*) \\ Département Génie Civil, I.U.T. de Saint-Nazaire, BP 420, 44606 Saint-Nazaire, France
}

(Reçu le 20 mai 1988, révisé et accepté le 27 juillet 1989)

\begin{abstract}
Résumé. - On propose ici une méthode non destructive de détermination des caractéristiques thermophysiques d'un matériau plastique prélevé par carottage ou d'un matériau obtenu par coulage. Un film chauffant est appliqué sur la face latérale de la carotte et les caractéristiques sont déduites de l'évolution des températures superficielle et axiale. Une étude théorique a été menée et un dispositif expérimental très simple a permis de corroborer ces considérations théoriques et mis en évidence les risques, dans certaines conditions, de limitation du procédé.
\end{abstract}

\begin{abstract}
Our purpose is to describe a non-destructive method to determine the thermophysical properties of a plastic material which was taken by tube sample coring or of a poured and formed material. A heating sheet is laid on the lateral side of the test cylinder and the characteristics may be deduced from the evolution of superficial and axial temperatures. A theoretical study has been conducted and a very simple experimental device has enabled us to confirm these theoretical conjectures and the conditions under which there exists a risk for the procedure may be partially limited.
\end{abstract}

\section{Introduction.}

Le présent article, comme deux précédents $[1,2]$, est consacré à la détermination dynamique des propriétés thermophysiques de matériaux du Génie Civil. Ces articles constituent une partie d'un travail en cours relatif aux transferts couplés de chaleur et d'humidité au sein de matériaux poreux - de premiers éléments d'approche du transfert isotherme d'humidité ont été donnés en [3] -

Nous nous sommes intéressé en premier à des matériaux relativement plastiques (en l'occurrence des argiles d'estuaire) prélevés par carottage. Les propriétés mécaniques des échantillons ainsi que le mode de prélèvement amènent à concevoir une méthode d'investigation appropriée : respect de la géométrie initiale, non-destructivité, et même, absence de perturbation génératrice de transferts d'humidité.

$\left(^{*}\right)$ Chercheur rattaché au Laboratoire de Génie Civil de l'E.N.S.M. de Nantes.
On trouvera des éléments de bibliographie sur la métrologie des propriétés thermophysiques des matériaux notamment chez Pratt [4], Mac Elroy et Moore [5] et Leidenfrost [6]. Farouki [7] a également énuméré divers procédés de mesure, en laboratoire ou in situ, de ces propriétés en se limitant au cas des sols. En suivant sa classification on distinguera :

- en régime statique : plaque chaude gardée, montages à symétrie cylindrique [5], sphère chauffante (Mochlinski [8]), mesure de flux entre deux points du sol (Van Wijk et Bruijn [9]);

- en régime dynamique :

- sonde cylindrique (technique très souvent utilisée) : Van der Held et Van Drunen [10] l'ont appliquée aux liquides, Hooper et Lepper [11] puis De Vries [12] et Buettner [13] aux sols, Destable [14] à des matériaux pulvérulents ;

- éprouvette cylindrique soumise latéralement, soit à une température variant sinusoïdalement, soit à un choc thermique, l'évolution de la température étant observée sur l'axe (Shannon et Wells [15], Mitchell et Kao [16]). 
C'est une variante de ce dernier procédé que nous nous proposons d'exposer. L'étude, voisine de [2] présente certains traits spécifiques liés à la géométrie du problème traité et à l'usage qu'on peut en faire.

\section{Etude théorique.}

2.1 MODÈLE DE BASE. - Le dispositif expérimental décrit plus loin en 3 peut être modélisé ainsi : un film chauffant délivrant une densité de flux $P$, infiniment mince, de capacité calorifique négligeable, entoure avec un contact parfait un cylindre circulaire de rayon $R$ d'un matériau homogène et isotrope caractérisé par sa diffusivité $a$ et sa conductivité $\lambda$; la chaleur volumique est $\rho c=\frac{\lambda}{a}$ et l'effusivité (ou inertie thermique) $b=\sqrt{\lambda \rho c}$. Film et cylindre sont entourés par un isolant parfait ; le cylindre est de longueur infinie ou, ce qui revient au même, de longueur finie avec ses bases parfaitement isolées.

L'évolution de la température $\theta(r, t)$ à la distance $r$ de l'axe est décrite selon Carslaw et Jaeger [17] par :

$$
\begin{aligned}
\theta(r, t)=\frac{P R}{\lambda}\left[\frac{2 a t}{R^{2}}+\frac{r^{2}}{2 R^{2}}\right. & -\frac{1}{4}-2 \times \\
& \left.\times \sum_{s=1}^{\infty} \mathrm{e}^{-\frac{a \alpha_{s}^{2} t}{R^{2}}} \frac{J_{0}\left(\alpha_{s} r / R\right)}{\alpha_{s}^{2} J_{0}\left(\alpha_{s}\right)}\right]
\end{aligned}
$$

avec les $\alpha_{s}$ racines positives de l'équation $J_{1}(\alpha)=0$; on limitera en fait la série à ses six premiers termes ce qui est très suffisant sauf lorsque $t$ tend vers zéro. Afin d'obtenir une formulation plus générale, adimensionnelle, on pose :

$$
\theta^{*}=\frac{\lambda \theta}{P R}, \quad t^{*}=\frac{a t}{R^{2}}
$$

d'où pour $r=R$ et $r=0$ respectivement :

$$
\begin{aligned}
& r=R, \theta_{1}^{*}=2 t^{*}+\frac{1}{4}-2 \sum_{s=1}^{6} \frac{\mathrm{e}^{-\alpha_{s}^{2} t^{*}}}{\alpha_{s}^{2}} \\
& r=0, \theta_{2}^{*}=2 t^{*}-\frac{1}{4}-2 \sum_{s=1}^{6} \frac{\mathrm{e}^{-\alpha_{s}^{2} t^{*}}}{\alpha_{s}^{2} J_{0}\left(\alpha_{s}\right)} .
\end{aligned}
$$

Remarque : On pourrait aussi poser : $t^{*}=\frac{a \alpha_{1}^{2} t}{R^{2}}$ ce qui aurait l'avantage de mettre en évidence la constante de temps: $\tau=\frac{R^{2}}{a \alpha_{1}^{2}}$ mais amène par contre à des expressions plus lourdes.

$\mathrm{Si}$ on fait d'abord suivre la transformation de Laplace d'un développement en série des fonctions de Bessel $I_{0}$ et $I_{1}$ en fonction de $1 / q$ et si on effectue alors la transformation inverse, on obtient une solution intéressante lorsque $r / R$ n'est pas petit

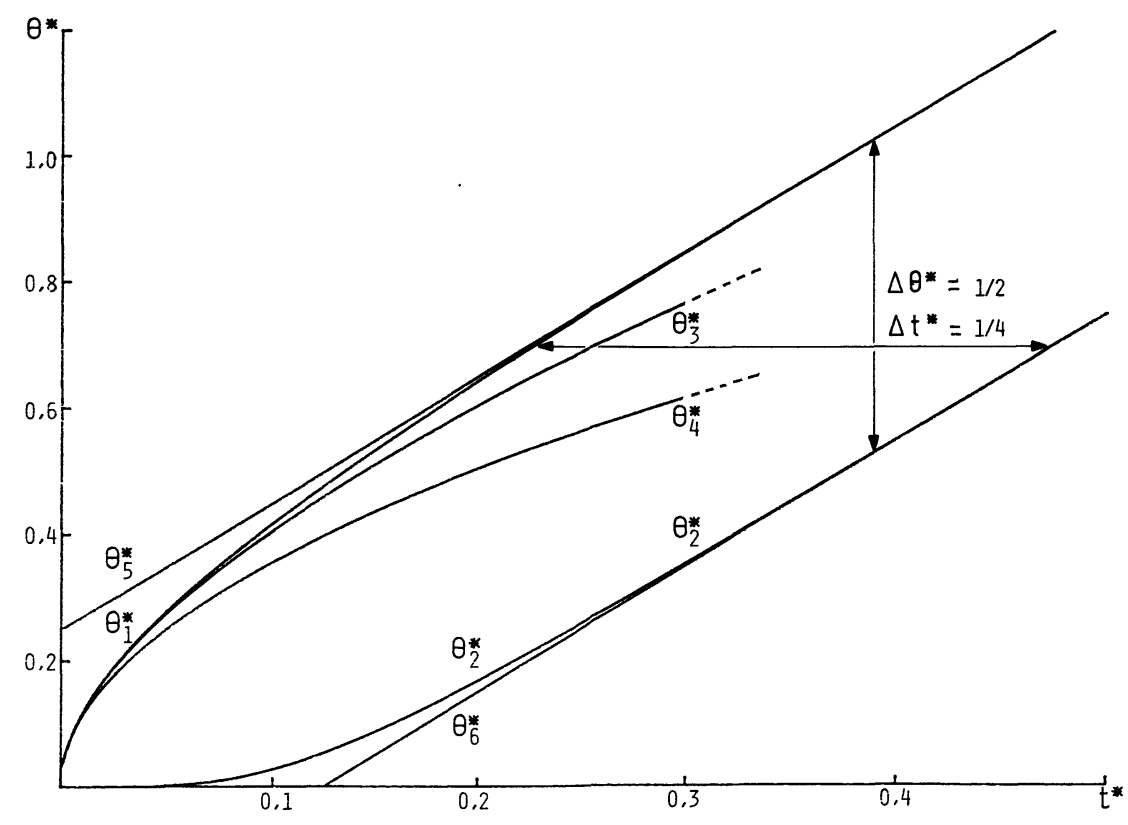

Fig. 1. - Températures $\theta^{*}$ en fonction de $t^{*}: \theta_{1}^{*}:$ surface $(r=R), \theta_{2}^{*}:$ axe $(r=0) ; \theta_{3}^{*}$ et $\theta_{4}^{*}:$ approximations de $\theta_{1}^{*}$ quand $t^{*} \rightarrow 0 ; \theta_{5}{ }^{*}$ et $\theta_{6}^{*}$ : asymptotes de $\theta_{1}^{*}$ et $\theta_{2}^{*}$.

[Temperatures $\theta^{*}$ versus $t^{*}: \theta_{1}^{*}$ : surface $(r=R) ; \theta_{2}^{*}:$ axis $(r=0) ; \theta_{3}^{*}$ and $\theta_{4}^{*}$ : approximations of $\theta_{1}^{*}$ when $t^{*} \rightarrow 0 ; \theta_{5}^{*}$ and $\theta_{6}^{*}$ : asymptotes of $\theta_{1}^{*}$ and $\theta_{2}^{*}$.] 
[17] ; en particulier, pour $r=R$ en se limitant aux deux premiers termes du développement :

$$
\theta_{3}^{*}=2 \sqrt{t^{*}} i \operatorname{erfc} 0+2 t^{*} i^{2} \operatorname{erfc} 0
$$

soit :

$$
r=R, \quad \theta_{3}^{*}=\frac{2}{\sqrt{\pi}} \sqrt{t^{*}}+\frac{t^{*}}{2}
$$

on a représenté graphiquement (Fig. 1) $\theta_{1}^{*}, \theta_{2}^{*}$, $\theta_{3}^{*}$ en fonction de $t^{*}$ ainsi que :

$r=R, \quad\left\{\begin{array}{l}\theta_{4}^{*}=\frac{2}{\sqrt{\pi}} \sqrt{t^{*}} \\ \theta_{5}^{*}=2 t^{*}+\frac{1}{4} \\ \theta_{6}^{*}=2 t^{*}-\frac{1}{4} .\end{array}\right.$

On a représenté également $\theta_{1}^{*}, \theta_{3}^{*}, \theta_{4}^{*}$ en fonction de $\sqrt{t^{*}}$ (Fig. 2).

2.2 EXPLOITATION DU MODÈLE. - On distinguera trois domaines d'étude : $t^{*}$ grand, court, moyen.

2.2.1. - $\theta_{1}^{*}$ et $\theta_{2}^{*}$ sont assimilables respectivement aux droites $\theta_{5}^{*}$ et $\theta_{6}^{*}$ à 0,01 près dès que $t^{*}>$ 0,17 et $t^{*}>0,24$. Au-delà de cette seconde limite on aura : $\Delta \theta^{*} \equiv \theta_{1}^{*}-\theta_{2}^{*}=1 / 2$ soit en revenant à la formulation initiale :

$$
\lambda=\frac{P R}{2 \Delta \theta} .
$$

On mettra de même en évidence $\Delta t^{*}=1 / 4$, d'où :

$$
\begin{aligned}
& a=\frac{R^{2}}{4 \Delta t} \\
& \rho c=\frac{2 P \Delta t}{R \Delta \theta} \\
& b=\frac{P \Delta(\sqrt{t})}{\Delta \theta} .
\end{aligned}
$$

2.2.2. - On considère ici l'écart relatif $\left(\theta_{1}^{*}-\theta_{4}^{*}\right) / \theta_{4}^{*}$. Cet écart est inférieur à un pour cent pour $\sqrt{t^{*}}<0,024$. En deçà de cette limite, on pourra écrire :

$$
b=\frac{2 P \Delta(\sqrt{t})}{\sqrt{\pi} \Delta \theta}
$$

2.2.3. - L'écart $\left(\theta_{1}^{*}-\theta_{3}^{*}\right) / \theta_{3}^{*}$ est inférieur à un pour cent pour $\sqrt{t^{*}}<0,2$; on pourra alors écrire :

$$
r=R, \quad \theta_{1}=P\left[\frac{2 \sqrt{t}}{b \sqrt{\pi}}+\frac{t}{2 \operatorname{R\rho c}}\right] .
$$

Ayant déterminé $b$ par (11) on obtiendra (Fig. 3) :

$$
\rho c=\frac{P \Delta t}{2 R \Delta\left[\theta_{1}-\frac{2 P \sqrt{t}}{b \sqrt{\pi}}\right]} .
$$

2.2.4. - Faisant apparaître la coupure du chauffage à l'instant $t_{\mathrm{c}}$ comme résultant de la superposition des

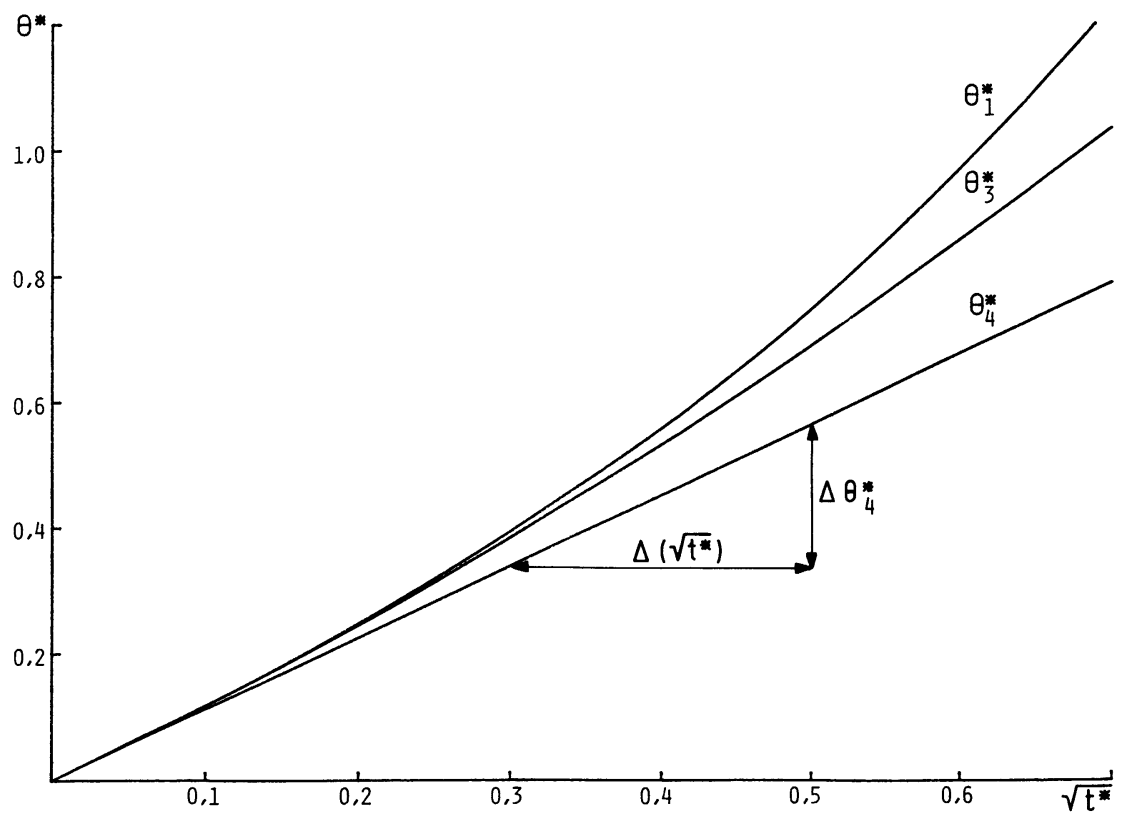

Fig. 2. - Température superficielle $\theta_{1}^{*}(r=R)$ et ses approximations $\theta_{3}^{*}$ et $\theta_{4}^{*}$ en fonction de $\sqrt{t^{*}}$.

[Superficial temperature $\theta_{1}^{*}(r=R)$ and its approximations $\theta_{3}^{*}$ and $\theta_{4}^{*}$ versus $\sqrt{t^{*}}$.] 


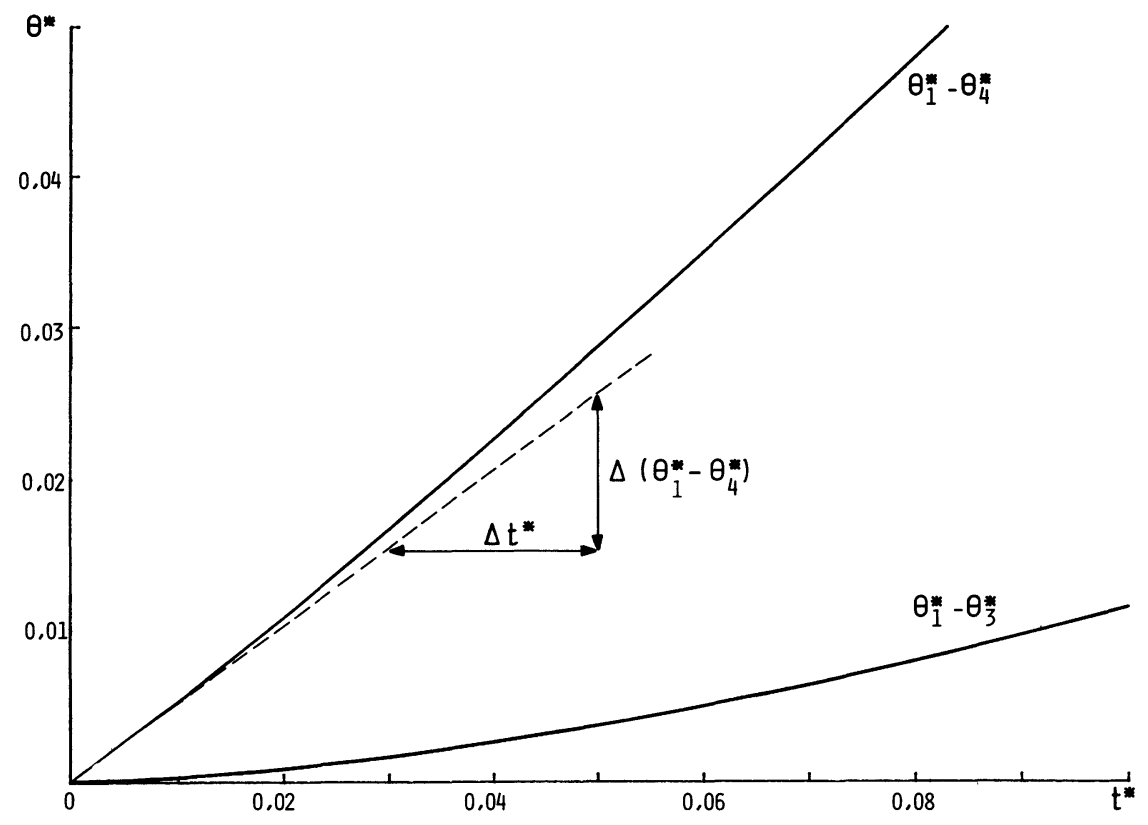

Fig. 3. - Ecart entre valeurs approchées $\theta_{3}^{*}$ et $\theta_{4}^{*}$ et exacte $\theta_{1}^{*}$ de la température superficielle $(r=R)$ en fonction de $t *$.

[Difference between approached values $\theta_{3}{ }^{*}$ and $\theta_{4}^{*}$ and true value $\theta_{1}^{*}$ of the superficial temperature $(r=R)$ versus $t^{*}$.]

sources $+P$ depuis $t=0$ et $-P$ depuis $t=t_{\mathrm{c}}$ on écrira à partir de l'expression de $\theta_{1}^{*}$ :

$$
\begin{aligned}
r=R, \quad \theta_{1}^{\prime *} & \equiv \theta_{1}^{*}+\theta_{1}^{*}= \\
& =2 t_{\mathrm{c}}^{*}-2 \sum_{s=1}^{6} \frac{1}{\alpha_{s}^{2}}\left(\mathrm{e}^{-\alpha_{s}^{2} t^{*}}-\mathrm{e}^{-\alpha_{s}^{2}\left(t^{*}-t_{\mathrm{c}}^{*}\right)}\right)
\end{aligned}
$$

et à partir de l'expression de $\theta_{4}^{*}$ :

$r=R, \quad \theta_{4}^{\prime *} \equiv \theta_{4}^{*}+\theta_{4}^{\prime *}=\frac{2}{\sqrt{\pi}}\left(\sqrt{t^{*}}-\sqrt{t^{*}-t_{\mathrm{c}}^{*}}\right)$.

Représentant $\theta_{1}^{\prime *}$ en fonction de $\left(\sqrt{t^{*}}-\sqrt{t^{*}-t_{\mathrm{c}}^{*}}\right)$ on voit que $\left(t^{*}-t_{\mathrm{c}}^{*}\right)$ petit amène à l'obtention de $b$ (Fig. 4) :

$$
b=\frac{2 P \Delta\left(\sqrt{t}-\sqrt{t-t_{\mathrm{c}}}\right)}{\sqrt{\pi} \Delta \theta}
$$

et que : $\theta_{\infty}^{\prime \prime *} \equiv \lim \theta_{1}^{\prime *}=2 t_{\mathrm{c}}^{*}$ amène à :

$$
\rho c=\frac{2 P t_{\mathrm{c}}}{R \theta_{\infty}^{\prime \prime}} \text {. }
$$

2.2.5. - Si on chauffe simultanément avec un même film deux éprouvettes $\mathrm{A}$ et $\mathrm{B}$ de même rayon, l'une formée d'un matériau de référence de caractéristiques stables, l'autre étant la carotte à étudier et si on effectue un enregistrement $X$ - $Y$ des températures superficielles $\theta_{1 \mathrm{~A}}$ et $\theta_{1 \mathrm{~B}}$ on obtient pour les temps courts d'après (11) :

$$
b_{\mathrm{B}}=b_{\mathrm{A}} \frac{\Delta \theta_{1 \mathrm{~A}}}{\Delta \theta_{1 \mathrm{~B}}}
$$

et pour les temps longs d'après (9) :

$$
(\rho c)_{\mathrm{B}}=(\rho c)_{\mathrm{A}} \frac{\Delta \theta_{1 \mathrm{~A}}}{\Delta \theta_{1 \mathrm{~B}}} .
$$

2.3 AfFINEMENT DU MODÈLE DE BASE. - Diverses erreurs systématiques ont été analysées séparément en [2] : isolation imparfaite du montage, effet capacitif du film, effet résistif d'une lame d'air entre film et éprouvette ou de la pellicule protectrice du conducteur. On a adopté ici une démarche convenant particulièrement aux temps courts : l'ensemble filmlame d'air est modélisé comme une capacité thermique surfacique $C$ suivie d'une résistance thermique d'épaisseur e et de conductivité $\lambda_{2}$. Dans la mesure où on s'intéresse à la température superficielle de la carotte et pour des temps courts on est fondé (dans un premier temps) à considérer $R$ comme infini. On 


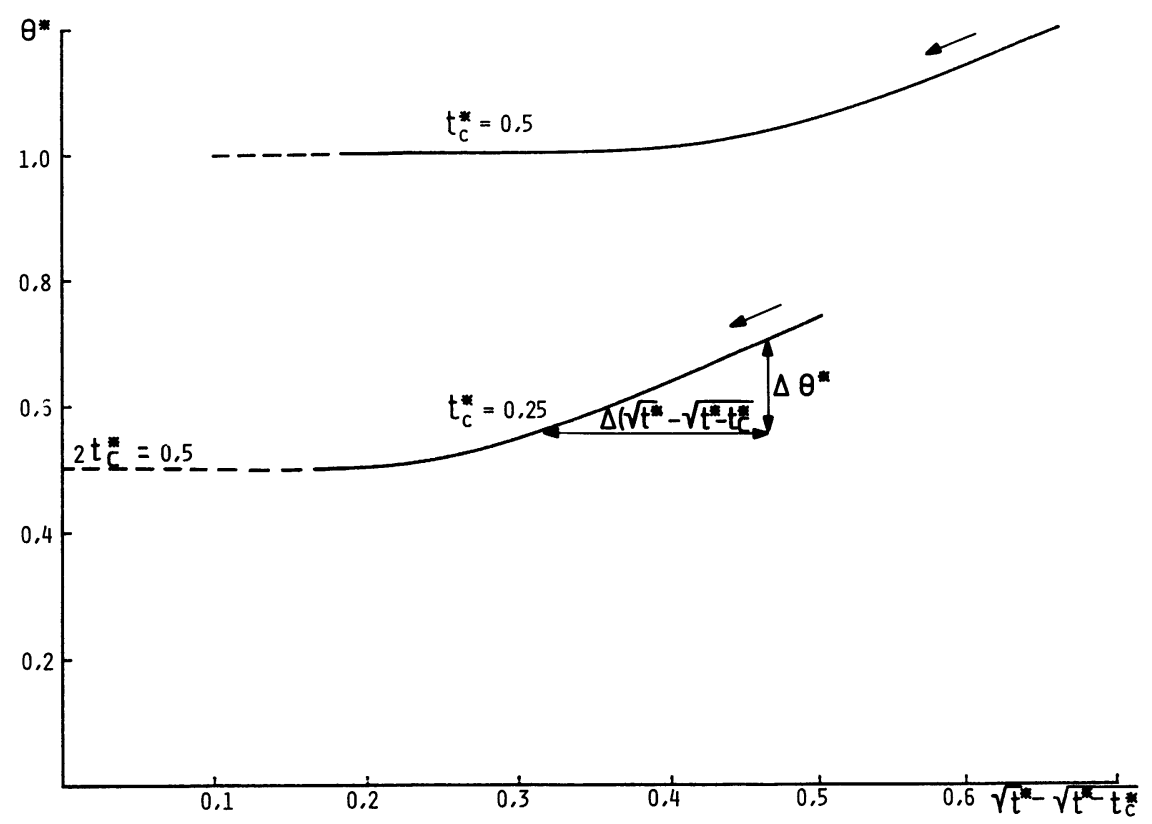

Fig. 4. - Réponse à un créneau de chauffage de durée $t_{\mathrm{c}}^{*}:$ température superficielle $\theta^{*}(r=R)$ en fonction de $\left(\sqrt{t^{*}}-\sqrt{t^{*}-t_{\mathrm{c}}^{*}}\right)$.

[Response to a heating rectangular pulse : superficial temperature $\theta^{*}(r=R)$ versus $\left(\sqrt{t^{*}}-\sqrt{t^{*}-t_{\mathrm{c}}^{*}}\right)$.]

est ramené à un problème plan avec milieu semiinfini. On a le système :

$$
\begin{cases}\frac{\partial^{2} \theta_{1}}{\partial x^{2}}-\frac{1}{a_{1}} \frac{\partial \theta_{1}}{\partial t}=0, & x \in[0,+\infty[ \\ \frac{\partial^{2} \theta_{2}}{\partial x_{2}}=0, & x \in[-e, 0[ \\ -\lambda_{2} \frac{\partial \theta_{2}}{\partial x}=P-C \frac{\partial \theta_{2}}{\partial t}, & x=-e \\ -\lambda_{2} \frac{\partial \theta_{2}}{\partial \chi}=-\lambda, \frac{\partial \theta_{1}}{\partial \chi}, & x=0 \\ \theta_{1}=\theta_{2}, & x=0 \\ \theta_{1}=\theta_{2}=0, & \forall x, t \leqslant 0 .\end{cases}
$$

Effectuant la transformation de Laplace, on cherche des solutions de la forme :

$$
\begin{aligned}
& \bar{\theta}_{1}=A_{1} \mathrm{e}^{-q_{1} x} \\
& \bar{\theta}_{2}=A_{2} x+B_{2} .
\end{aligned}
$$

$A_{1}, A_{2}, B_{2}$ sont déterminés à partir de l'expression des conditions aux limites; on a en particulier :

$$
\begin{aligned}
\bar{\theta}_{0} \equiv\left(\bar{\theta}_{1}\right)_{x=0}=\frac{P \lambda_{2}}{b_{1} C e} \cdot \frac{1}{p^{5 / 2}} & \times \\
& \times \frac{1}{1+\frac{\lambda_{2}}{b_{1} e} \cdot \frac{1}{p^{5 / 2}}+\frac{\lambda_{2}}{C e} \cdot \frac{1}{p}} .
\end{aligned}
$$

Pour $p$ grand et donc $t$ petit on effectue un développement limité en $1 / p$ :

$$
\begin{aligned}
\bar{\theta}_{0}=\frac{P \lambda_{2}}{b_{1} C e}\left[\frac{1}{p^{5 / 2}}-\right. & \frac{\lambda_{2}}{b_{1} e} \cdot \frac{1}{p^{3}}+ \\
& \left.+\left(\frac{\lambda_{2}^{2}}{b_{1}^{2} e^{2}}-\frac{\lambda_{2}}{C e}\right) \cdot \frac{1}{p^{7 / 2}}+\cdots\right] .
\end{aligned}
$$

Par transformation inverse et avec la mise sous forme adimensionnelle suivante :

$$
\lambda *=\frac{\lambda_{2}}{\lambda_{1}}, \quad \ell *=\frac{e}{R}, \quad C *=\frac{C}{\rho_{1} c_{1} R}
$$

on obtient pour $x=0$ :

$$
\begin{aligned}
& \theta_{0}^{*}=\frac{4}{3 \sqrt{\pi}} \frac{\lambda^{*}}{\ell^{*} C^{*}} t^{* 3 / 2}-\frac{1}{2} \frac{\lambda^{* 2}}{\ell^{* 2} C^{*}} t^{* 2}+ \\
& +\frac{8}{15 \sqrt{\pi}}\left(\frac{\lambda^{* 3}}{\ell^{* 3} C^{*}}-\frac{\lambda^{* 2}}{\ell^{* 2} C^{* 2}}\right) t^{* 5 / 2}+\cdots
\end{aligned}
$$

On obtiendrait de même pour $x=-e$ :

$$
\begin{aligned}
\theta_{-e}^{*}=\theta_{0}^{*} & +\frac{t^{*}}{C^{*}}-\frac{4}{3 \sqrt{\pi}} \frac{\lambda^{*}}{\ell * C^{*}} t^{* 3 / 2}+ \\
& +\left(\frac{\lambda^{* 2}}{\ell^{* 2} C^{*}} \frac{\lambda^{*}}{\ell * C^{* 2}}\right) \frac{t^{* 2}}{2}+\cdots
\end{aligned}
$$


Pour $p$ petit et donc $t$ grand un développement limité en $p$ donne :

$\theta_{0}=\frac{P}{b_{1}}\left[2 \sqrt{\frac{t}{\pi}}-\frac{C}{b_{1}}+\left(\frac{C^{2}}{b_{1}^{2}}-\frac{C e}{\lambda_{2}}\right) \frac{1}{\sqrt{\pi t}}+\cdots\right]$.

D'où par les mêmes techniques :

$$
\begin{gathered}
\theta_{0}^{*}=2 \sqrt{\frac{t^{*}}{\pi}}-C^{*}+\left(C^{* 2}-\frac{\ell * C^{*}}{\lambda^{*}}\right) \frac{1}{\sqrt{\pi t^{*}}} \\
\theta_{-e}^{*}=\theta_{0}^{*}+\frac{\ell^{*}}{\lambda^{*}}\left(1-\frac{C^{*}}{\sqrt{\pi t^{*}}}\right) .
\end{gathered}
$$

Il faut noter que l'approximation $p$ petit, $t$ grand doit être utilisée avec précaution : on devra vérifier la validité de l'hypothèse du milieu semi-infini soit $\sqrt{t^{*}}<0,024$ comme vu en 2.2.2. A partir de (15), (16), (17), (18), on a tracé (Fig. 5) $\theta_{0}^{*}$ et $\theta_{-e}^{*}$ en fonction de $\sqrt{t^{*}}$ pour $\lambda^{*}=0,05, \ell^{*}=0,004$, $C^{*}=0,01$, ce qui correspond sensiblement à nos conditions expérimentales. Ces considérations ont été développées en [2]. On a tracé également (Fig. 6) $\theta^{*}$ en fonction de $\sqrt{t^{*}}$ pour un point intermédiaire de la résistance thermique, en l'occurrence $x=-e / 2$, soit :

$$
x=-\frac{e}{2}, \quad \theta_{7}^{*}=\frac{\theta_{0}^{*}+\theta_{-e}^{*}}{2}+\frac{t^{*}}{2}
$$

en prenant en compte le terme correctif $\frac{t^{*}}{2}$ qui permet de passer de $\theta_{4}^{*}$ à $\theta_{3}^{*}, \theta_{3}^{*}$ lui-même confondu avec $\theta_{1}^{*}$ sur l'intervalle $\sqrt{t^{*}} \in[0,0,024]$. On voit sur cet intervalle que les effets capacitif et résistif font que $\theta_{7}^{*}$ n'est pas une droite parallèle à $\theta_{4}^{*}$. On ne pourra envisager une mesure correcte de l'effusivité qu'en tentant de réduire $e$ au maximum car c'est la seule grandeur sur laquelle on puisse agir.

Le terme $\frac{t^{*}}{2}$ n'apparaissant pas dans le cas d'éprouvettes planes [2], les contraintes sur la mesure seraient, dans ce cas, beaucoup moins fortes.

Les démarches, séduisantes à première vue, décrites en 2.2.2 et en 2.2.3, sont donc à utiliser avec de grandes précautions.

\section{Etude expérimentale.}

3.1 DesCription du montage (Fig. 7). - Nous avons étudié tout d'abord un échantillon cylindrique de diamètre $10 \mathrm{~cm}$ et de longueur $15 \mathrm{~cm}$ prélevé dans une carotte d'argile de l'estuaire de la Loire, matériau soumis par ailleurs à de fréquentes investigations par Dupain [18], Dupain et Kismi [19].

Il s'agissait d'un type d'échantillon non remanié d'argile homogène sans passage sableux ; la cohésion peut varier pour ce matériau entre 10 et $35 \mathrm{kPa}$.

Un thermocouple est disposé sur l'axe après partition à l'aide d'un fil coupant de l'échantillon ; les deux moitiés de celui-ci réadhèrent ensuite sans difficulté. Un second thermocouple est disposé sur la paroi latérale. Deux demi-coquilles en mousse de

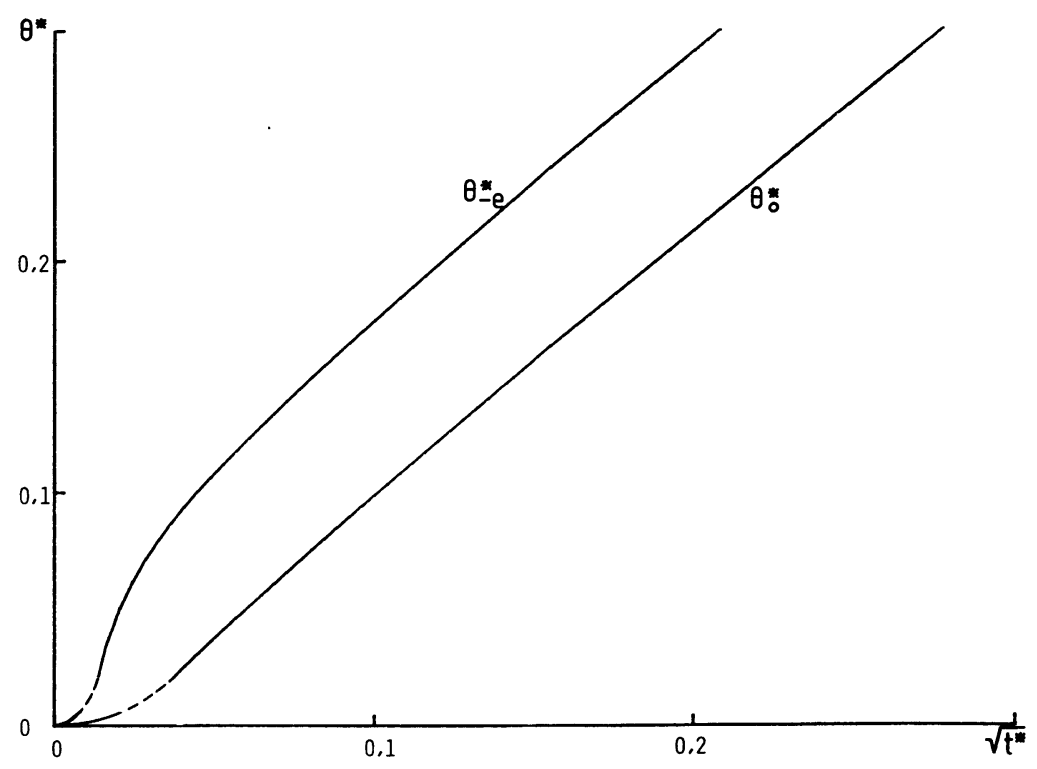

Fig. 5. - Températures superficielles $\theta_{-e}^{*}(r=R+e)$ : film, $\theta_{0}^{*}(r=R)$ : éprouvette en fonction de $\sqrt{t^{*}}$ (film chauffant capacitif suivi d'une résistance de contact).

[Superficial temperatures $\theta_{-e}^{*}(r=R+e)$ : sheet, $\theta_{0}^{*}(r=R)$ : test cylinder, versus $\sqrt{t^{*}}$ (capacitive heating sheet and contact-resistance).] 


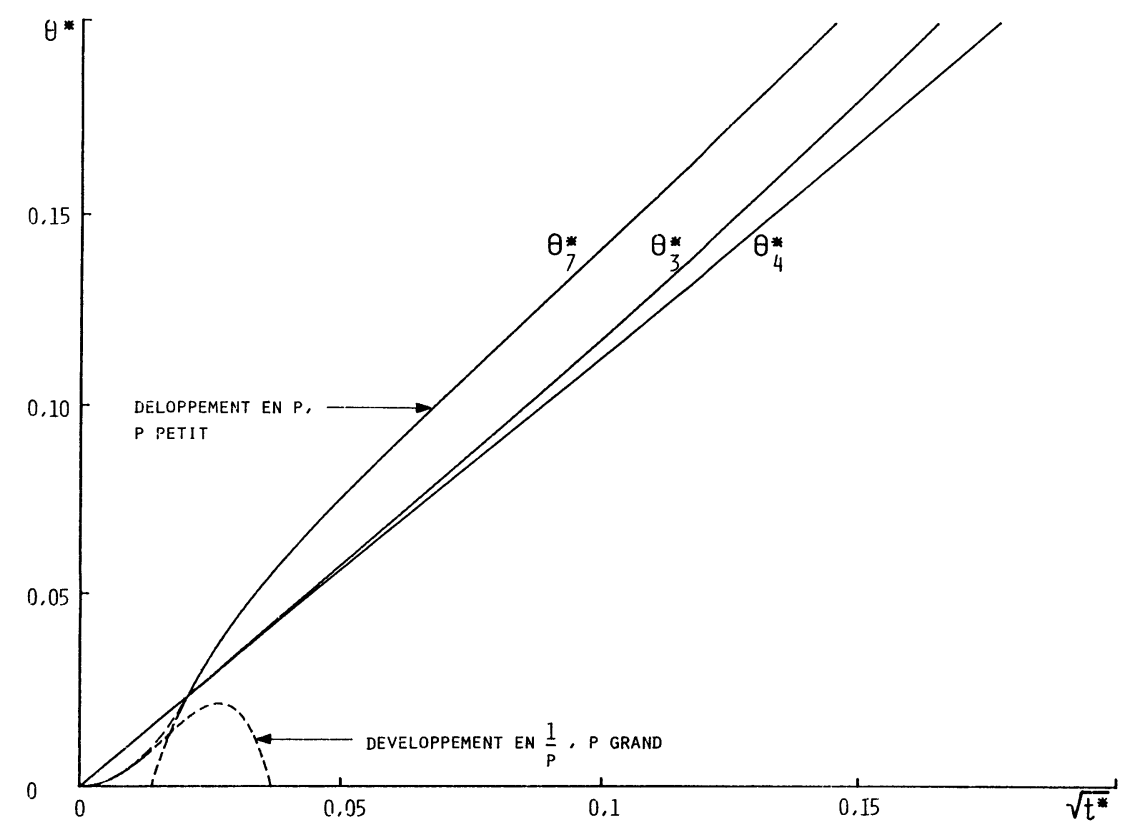

Fig. 6. - Approximations $\theta_{3}^{*}$ et $\theta_{4}^{*}$ de la température superficielle $(r=R)$. Température intermédiaire $\theta_{7}^{*}(r=$ $R+\frac{e}{2}$ ) (film chauffant capacitif suivi d'une résistance de contact).

$\left[\theta_{3}^{*}\right.$ and $\theta_{4}^{*}$ : approximations of the superficial temperature $(r=R) . \theta_{7}^{*}$ : intermediate temperature $(r=R+$ $\frac{e}{2}$ ) (capacitive heating sheet and contact-resistance).]

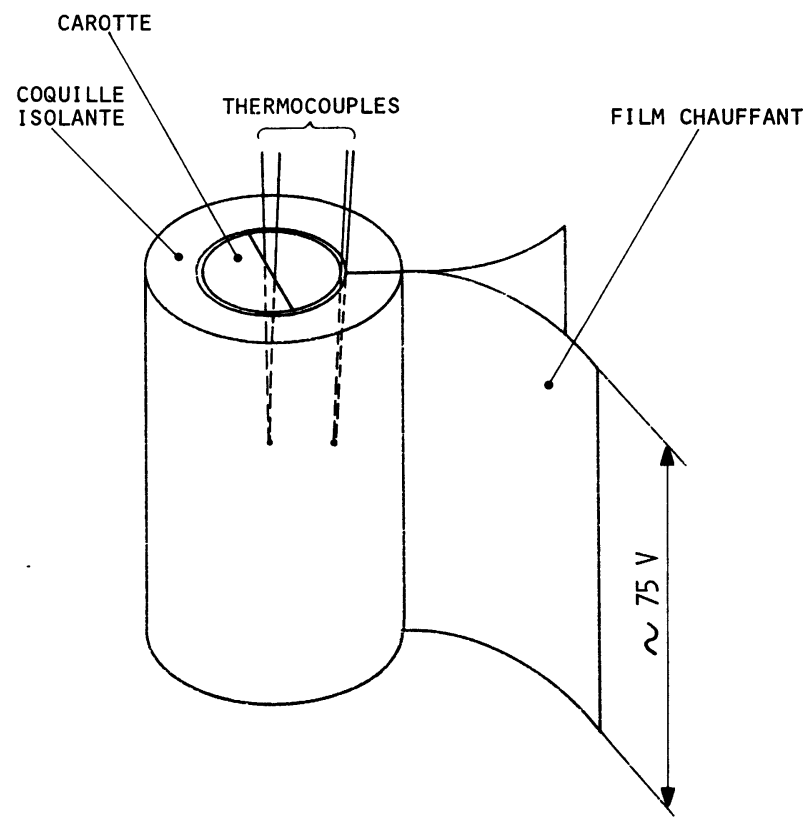

Fig. 7. - Montage expérimental.

[Experimental device.]

polyuréthane sont refermées sur le cylindre entouré préalablement d'un fil chauffant. Ce film de marque Semme est décrit plus précisément en [2] : il s'agit de rubans conducteurs non jointifs recouverts d'une pellicule isolante; on recouvre celle-ci de ruban adhésif en aluminium afin d'obtenir une densité de flux uniforme.

L'élément de film utilisé est alimenté sous une tension de l'ordre de $75 \mathrm{~V}$ qui est relevée ainsi que le courant traversant le film; la densité de flux est voisine de $200 \mathrm{~W} / \mathrm{m}^{2}$.

Les thermocouples sont reliés par l'intermédiaire d'une boîte de soudure froide à une chaîne voltmètre-microordinateur-table traçante.

\subsection{RÉSULTATS EXPÉRIMENTAUX POUR UN ÉCHAN- TILLON D'ARGILE.}

3.2.1. - On donne (Fig. 8) l'évolution des températures axiale et superficielle lors d'un chauffage de trente minutes. On constate qu'au bout de cette durée :

- la température de surface s'est élevée de plus de dix degrés, ce qui constitue un maximum admissible au-delà duquel il y a risque de voir évoluer les propriétés thermiques et mécaniques du matériau par migration d'humidité couplée au transfert de chaleur ;

- l'évolution des deux températures, et plus particulièrement celle de la température axiale (voir 2.2.1), n'est pas encore parfaitement linéaire.

On a effectué une extrapolation linéaire des 


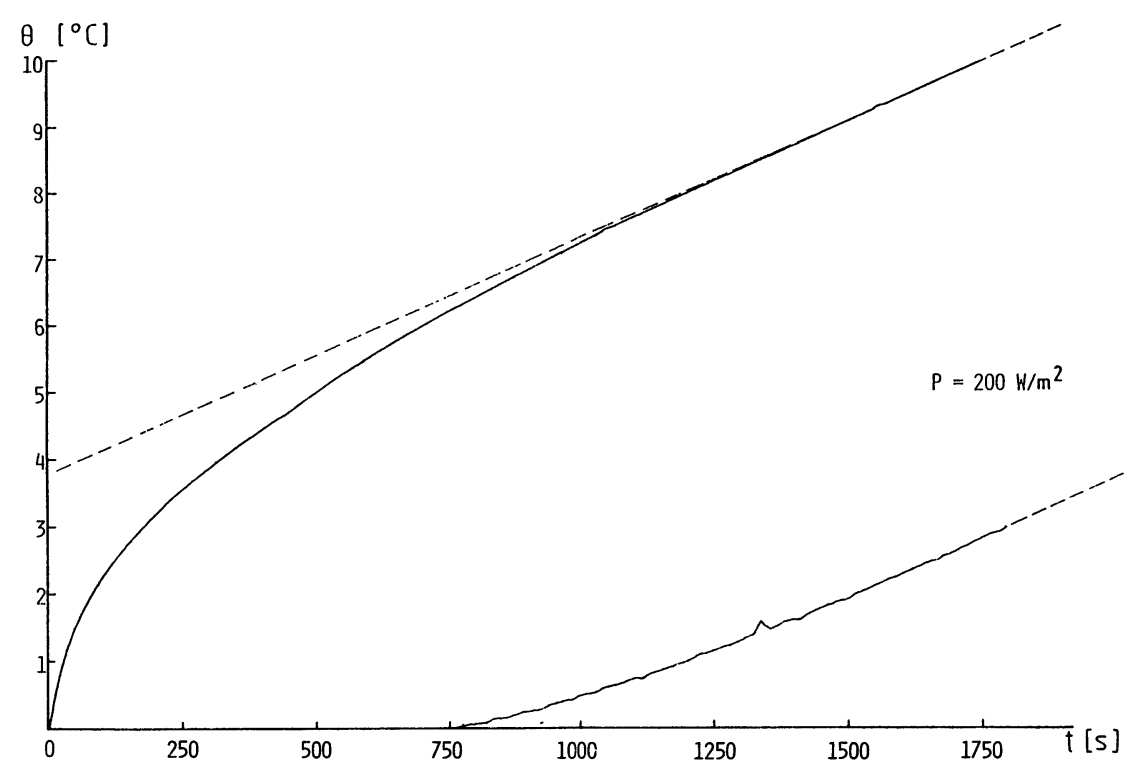

Fig. 8. - Argile : enregistrement d'une phase de chauffage. Températures superficielle et axiale en fonction du temps. [Clay : recording of a heating period. Superficial and axial temperatures versus time.]

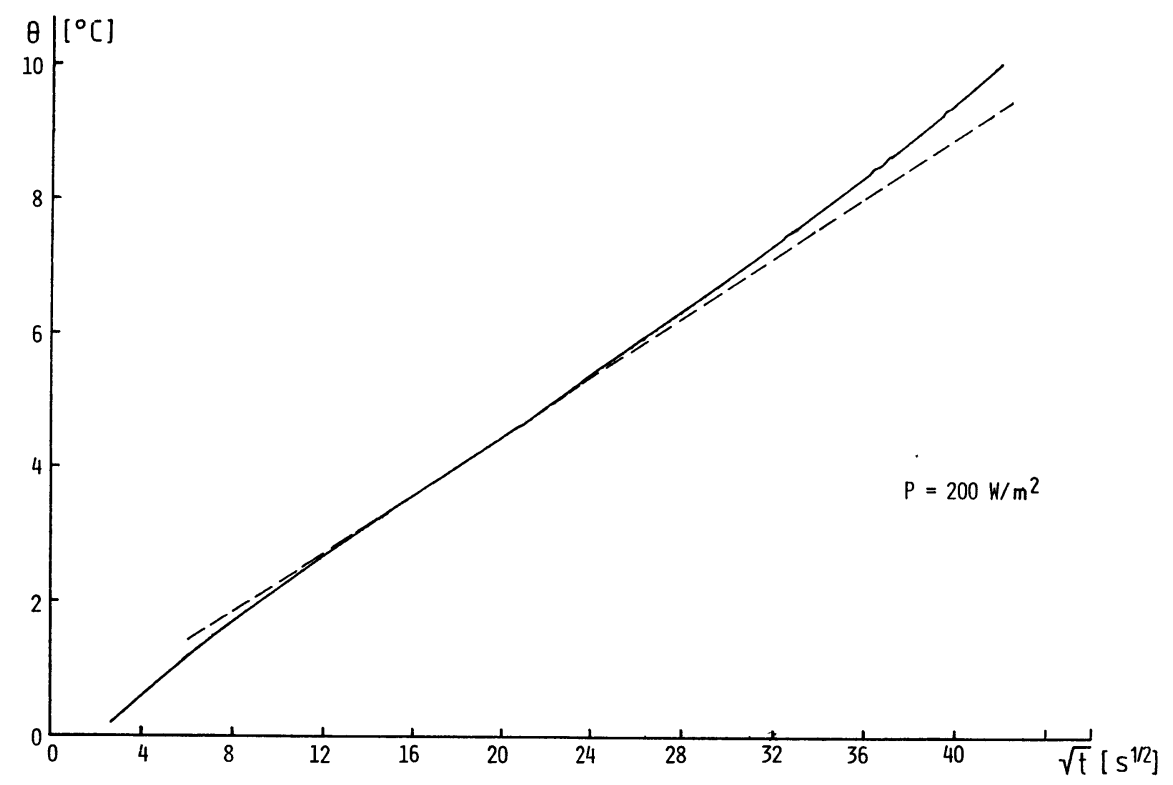

Fig. 9. - Argile : enregistrement d'une phase de chauffage. Température superficielle en fonction de $\sqrt{t}$. [Clay : recording of a heating period. Superficial temperature versus $\sqrt{t}$.]

températures superficielle et axiale et obtenu les résultats suivants :

$$
\begin{aligned}
\lambda & =0,69 \mathrm{~W} / \mathrm{m} \cdot \mathrm{K} \\
\rho c & =2,29 \times 10^{6} \mathrm{~J} / \mathrm{m}^{3} \cdot \mathrm{K} \\
a & \equiv \frac{\lambda}{\rho c}=3,01 \times 10^{-7} \mathrm{~m}^{2} / \mathrm{s} \\
b & \equiv \sqrt{\lambda \rho c}=1,26 \times 10^{3} \mathrm{~W} \cdot \mathrm{s}^{1 / 2} / \mathrm{m}^{2} \cdot \mathrm{K} .
\end{aligned}
$$

L'expression $t=\frac{R^{2}}{a} t^{*}$ jointe aux inégalités $t^{*}<$ 0,17 et $t^{*}<0,24$ nous montre qu'effectivement le régime linéaire n'est atteint qu'au bout d'une demiheure.

L'utilisation de (11) amène à $b=1,15 \times$ $10^{3} \mathrm{~W} . \mathrm{s}^{1 / 2} / \mathrm{m}^{2}$. K (Fig. 9). L'étude effectuée en 2.3 justifie cet écart, la valeur obtenue par (10) étant nettement plus fiable. 


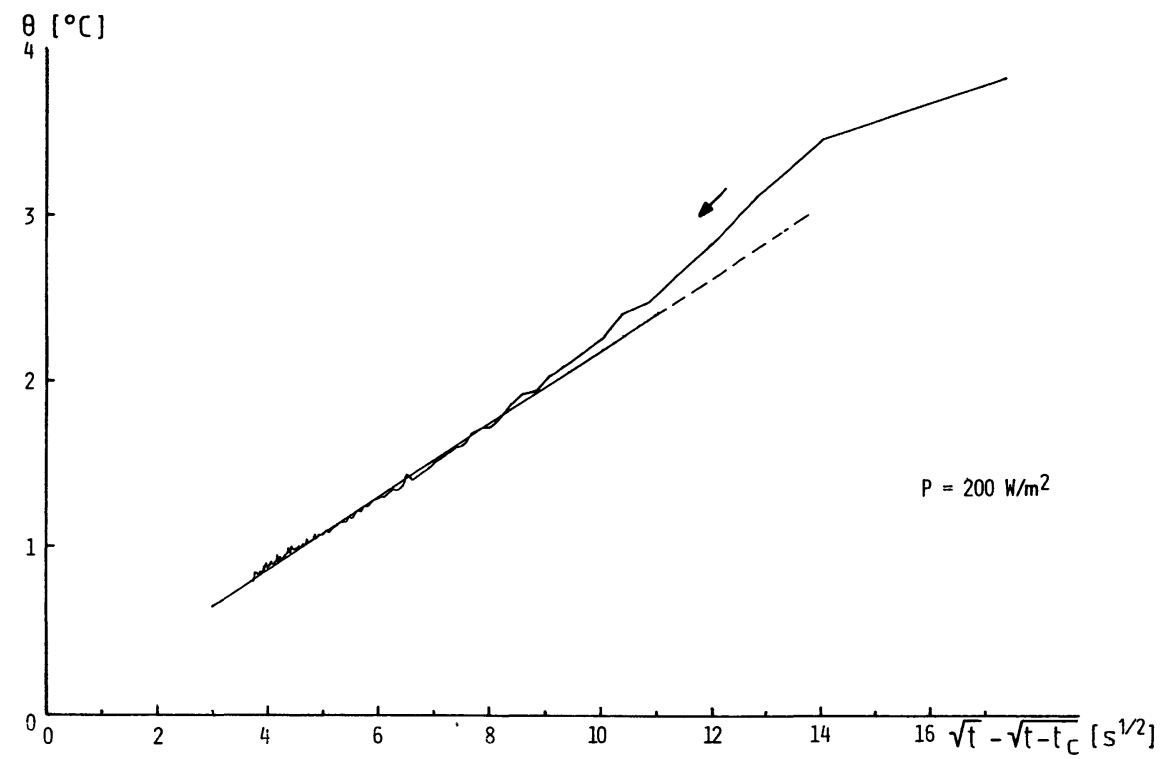

Fig. 10. - Argile : enregistrement de la réponse à un créneau de chauffage. Température superficielle en fonction de $\left(\sqrt{t}-\sqrt{t-t_{c}}\right)$.

[Clay : recording of a heating rectangular pulse response. Superficial temperature versus $\left(\sqrt{t}-\sqrt{t-t_{c}}\right)$.]

On a donné également (Fig. 10) la détermination de $b$ selon la démarche décrite en 2.2.4 mais elle est d'exploitation encore plus incertaine.

3.2.2. - On a remplacé le thermocouple fin placé axialement par un autre de fort diamètre faisant fonction de fil chaud émettant $P(\mathrm{~W} / \mathrm{m})$ suivant la méthode décrite en [1]. On dispose alors d'une seconde méthode de détermination de la conducti- vité (Fig. 11) ; on a ainsi trouvé :

$$
\lambda=0,70 \mathrm{~W} / \mathrm{m} \cdot \mathrm{K}
$$

à partir de la relation :

$$
\lambda=\frac{P}{4 \pi} \frac{\Delta \ln \frac{t}{t-t_{\mathrm{c}}}}{\Delta \theta}
$$

ce qui corrobore les résultats précédents.

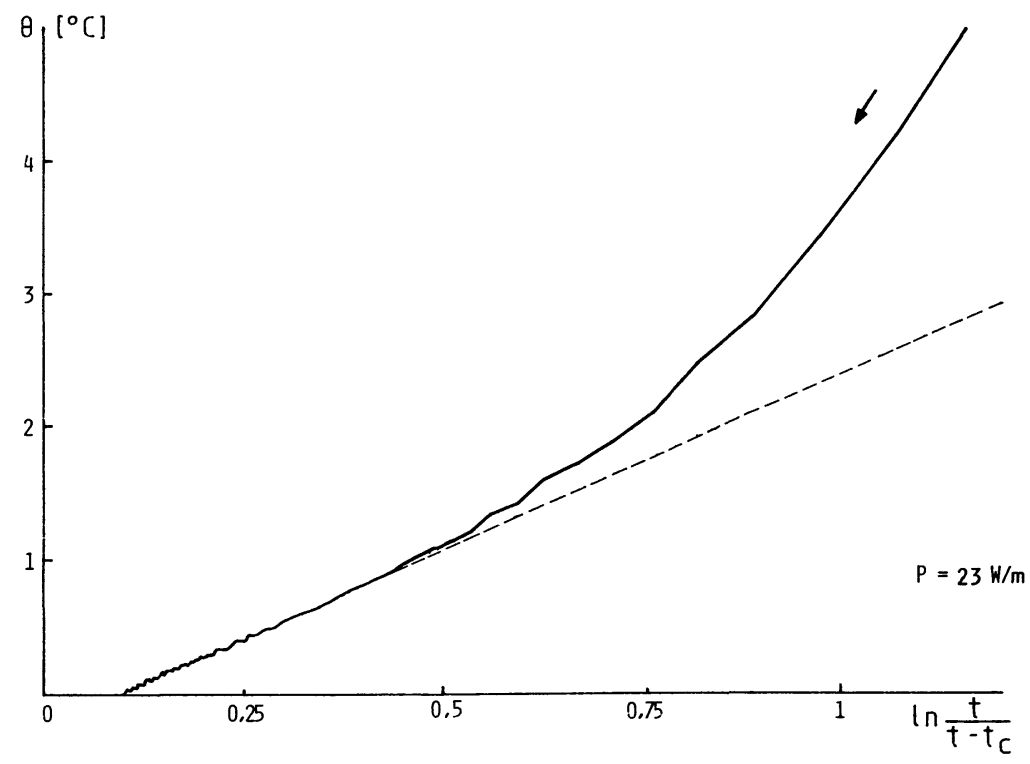

Fig. 11. - Argile : méthode du fil chaud. Enregistrement de la réponse à un créneau de chauffage. Température du fil en fonction de $\ln \frac{t}{t-t_{c}}$.

[Clay : hot wire method. Recording of a heating rectangular pulse response. Wire temperature versus $\left.\ln \frac{t}{t-t_{\mathrm{c}}}.\right]$ 


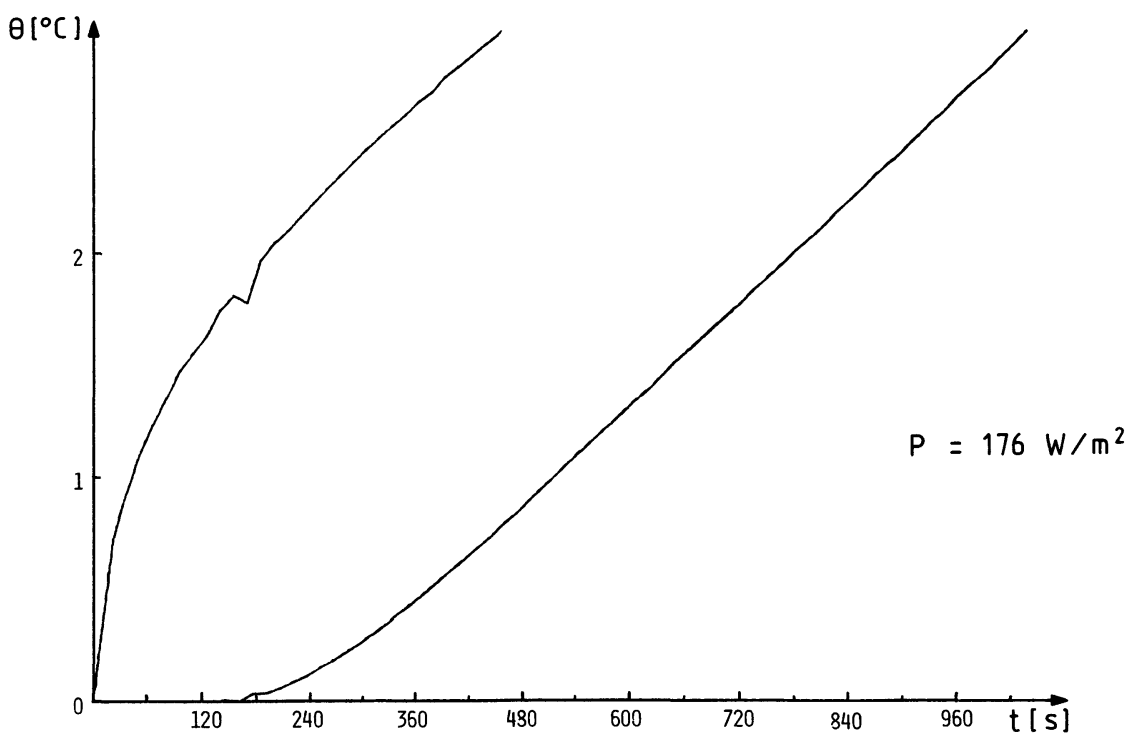

Fig. 12. - Mortier : enregistrement d'une phase de chauffage. Températures superficielle et axiale en fonction du temps.

[Mortar : recording of a heating period. Superficial and axial temperatures versus time.]

3.2.3. - L'opération de carottage proprement dite peut induire éventuellement des modifications superficielles des propriétés du matériau. Par contre, l'extraction de l'échantillon hors du tube de P.V.C. qui l'entoure se faisant par découpage à la meule du tube suivant deux génératrices opposées, ce procédé d'extraction ne crée pas de nouvelle perturbation. $\mathrm{Si}$ l'on éprouve quelque crainte quant à la fiabilité de mesures où l'on fait intervenir la température super- ficielle de la carotte on peut utiliser uniquement l'évolution de la température axiale pour déterminer les caractéristiques thermophysiques :

On a écrit en (6) : $\theta_{6}^{*}=2 t^{*}-\frac{1}{4}$ pour l'évolution asymptotique sur l'axe, soit :

$$
r=0, \quad \theta_{6}=\frac{2 P t}{\rho c R}-\frac{P R}{4 \lambda}
$$

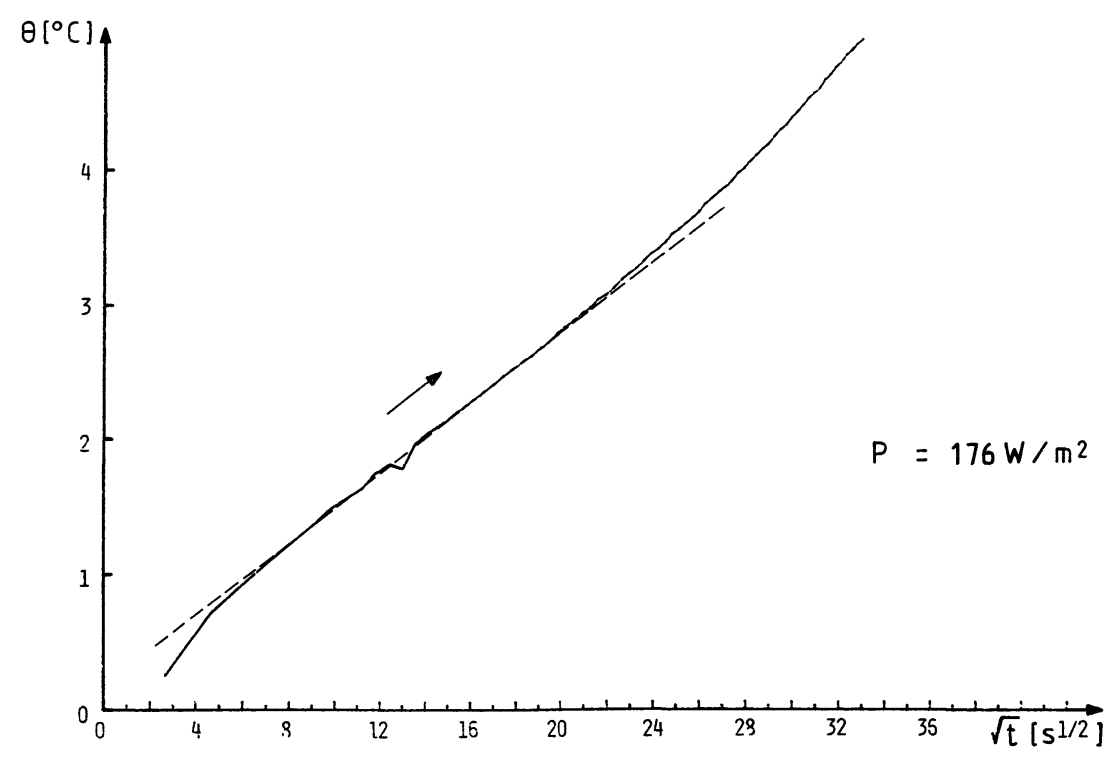

Fig. 13. - Mortier : enregistrement d'une phase de chauffage. Température superficielle en fonction de $\sqrt{t}$.

[Mortar : recording of a heating period. Superficial temperature versus $\sqrt{t}$.] 
D'où :

$$
\begin{aligned}
\lambda & =-\frac{P R}{4 \theta_{6}(0)}, & \theta_{6}(0) \equiv \theta_{6} \text { à } t=0 \\
\rho c & =\frac{2 P \Delta t}{R \Delta \theta_{6}}, & \frac{\Delta \theta_{6}}{\Delta t} \text { pente de } \theta_{6} \\
a & =\frac{R^{2}}{8 t_{0}}, & t_{0} \text { abscisse à } \theta_{6}=0
\end{aligned}
$$

on obtient ainsi :

$$
\begin{aligned}
\lambda & =0,72 \mathrm{~W} / \mathrm{m} \cdot \mathrm{K} \\
\rho c & =2,27 \times 10^{6} \mathrm{~J} / \mathrm{m}^{3} \cdot \mathrm{K} \\
a & =3,16 \times 10^{-7} \mathrm{~m}^{2} / \mathrm{s} .
\end{aligned}
$$

3.3 RÉSULTATS EXPÉRIMENTAUX POUR UN ÉCHANTILLON DE MORTIER. - Notre méthode de mesure a été ensuite appliquée à un mortier amené en équilibre hygrothermique avec l'ambiance du laboratoire ce qui conditionne sa teneur en eau. La longueur de l'éprouvette était $0,90 \mathrm{~m}$ et son diamètre $0,096 \mathrm{~m}$. Un fil chauffant capteur [1] avait été disposé axialement lors du couplage.

Composition et teneur en eau étaient identiques à celles d'éprouvettes étudiées en [2]. On a obtenu des résultats très voisins de ceux donnés en [2].

- méthode du film chaud :

- temps courts :

\begin{tabular}{c|c} 
film cylindrique & film plan [2] \\
\hline$b=1,49 \times 10^{3}$ & $b=1,72 \times 10^{3} \mathrm{~W} \cdot \mathrm{s}^{1 / 2} / \mathrm{m}^{2} \cdot \mathrm{K}$
\end{tabular}

- temps longs :

\begin{tabular}{c|c} 
film cylindrique & film plan [2] \\
\hline$\lambda=1,61$ & $\lambda=1,55 \mathrm{~W} / \mathrm{m} \cdot \mathrm{K}$ \\
$\rho c=1,97 \times 10^{6}$ & $\rho c=2,00 \times 10^{6} \mathrm{~J} / \mathrm{m}^{3} . \mathrm{K}$ \\
$a=0,82 \times 10^{-6}$ & $a=0,78 \times 10^{-6} \mathrm{~m}^{2} / \mathrm{s}$ \\
$b=1,78 \times 10^{3}$ & $b=1,78 \times 10^{3} \mathrm{~W} \cdot \mathrm{s}^{1 / 2} / \mathrm{m}^{2} . \mathrm{K}$
\end{tabular}

— méthode du fil chaud axial :

$$
\lambda=1,56 \mathrm{~W} / \mathrm{m} \cdot \mathrm{K} \text {. }
$$

Ainsi qu'on l'a déjà souligné à propos de la carotte d'argile la mesure directe de l'effusivité $b$ est à accueillir avec prudence. Il y a par contre une bonne corrélation entre les autres résultats.

\section{Conclusion.}

Nous avons décrit un montage simple, utilisable pour des matériaux plastiques qui, après partition, réadhèrent sans difficulté. Ce montage est également utilisable pour l'étude de matériaux obtenus par coulage - mortier notamment - le thermocouple axial étant incorporé lors de la phase de coulage.

Etudes théorique et expérimentale montrent qu'une durée de chauffage de plus d'une demi-heure peut être nécessaire pour obtenir des évolutions linéaires des températures; dans ces conditions il conviendra de réduire la densité de flux pour éviter

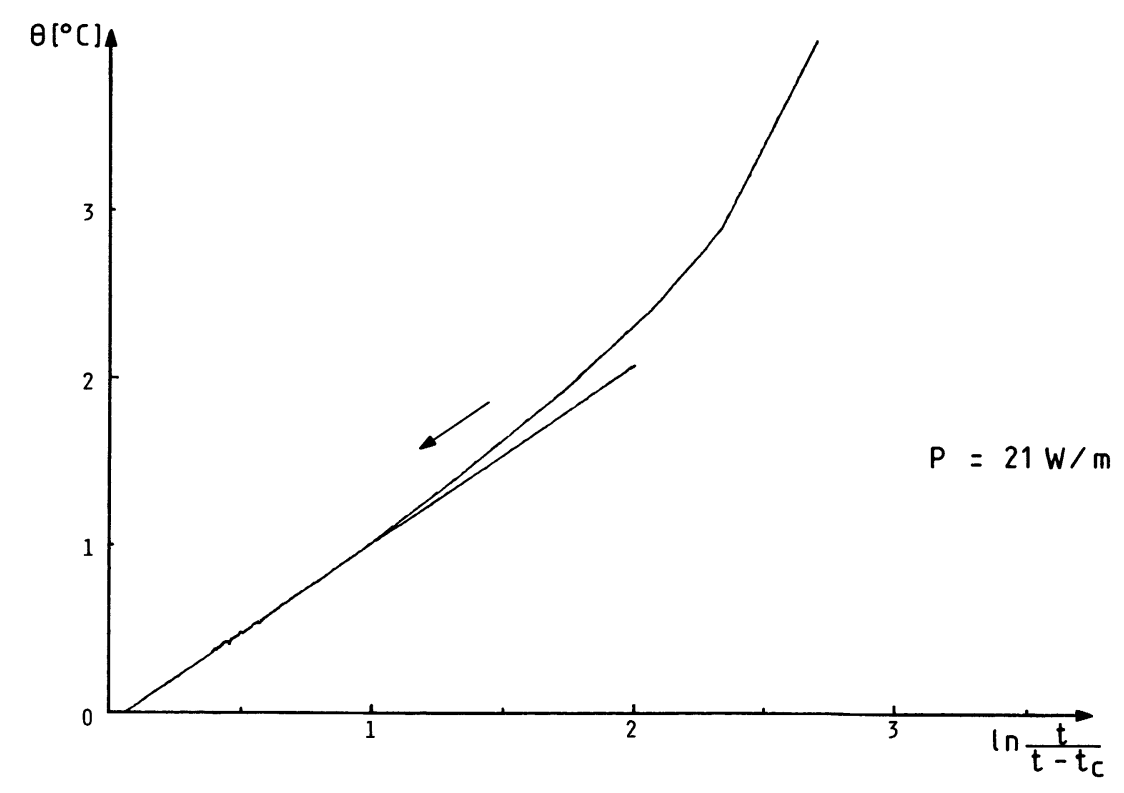

Fig. 14. - Mortier : méthode du fil chaud. Enregistrement de la réponse à un créneau de chauffage. Température du fil en fonction de $\ln \frac{t}{t-t_{\mathrm{c}}}$.

[Mortar : hot wire method. Recording of a heating rectangular pulse response. Wire temperature versus $\ln \frac{t}{t-t_{\mathrm{c}}}$.] 
des surchauffes trop importantes, sinon trop longues, génératrices de migration d'humidité.

Enfin, on a vu que la courbure de l'échantillon qui se traduit par l'existence d'un terme correctif $\frac{t^{*}}{2}$, terme qui n'existe pas dans le cas d'une éprouvette plane, jointe aux erreurs systématiques inhérentes au montage (effet capacitif, effet résistif plus ou moins important) peut rendre imprécise la mesure de l'effusivité.

Nous pensons que la méthode décrite en 3.2.1 sous réserve d'obtention d'un bon contact filméchantillon peut permettre d'obtenir les propriétés thermophysiques du matériau avec une précision relative de l'ordre de quatre à cinq pour cent.

\section{Bibliographie}

[1] BASTIAN G., Détermination de la conductivité thermique de matériaux de construction par une méthode transitoire type sonde cylindrique, Revue Phys. Appl. 17 (1982) 217.

[2] BASTIAN G., Détermination de caractéristiques thermophysiques de matériaux de construction par la méthode de la source plane en régimes transitoire et asymptotique, Revue Phys. Appl. 22 (1987) 431.

[3] BASTIAN G., Détermination de la conductivité massique d'humidité par une méthode transitoire de type sonde cylindrique (Groupement Universitaire de Thermique, Mesure de l'humidité dans les matériaux, Paris) 25/04/1984.

[4] Pratt A. W., Heat transmission in low conductivity materials, Thermal conductivity, Ed. R. P. Tye (Acad. Press, London and N.Y.) 1969.

[5] Mac Elroy D. L., Moore J. P., Radial heat flow methods for the measurement of the thermal conductivity of solids, Thermal conductivity, Ed. R. P. Tye (Acad. Press, London and N.Y.) 1969.

[6] LEIDENFROST W., Measurement of thermophysical properties, Measurements in heat transfer, Eds. R. G. Eckert, R. J. Goldstein (Hem. Pub. Corp., Wash.) 1976.

[7] Farouki O. T., Thermal properties of soils (Trans. Tech. Publications, Clausthall-Zellerfeld, R.F.A.) 1986.

[8] MOCHLINSKI K., Some industrial measurements of thermal properties of soils, Int. Study Group on Soils, Cambridge, England, 12-26/07/1964, p. 168.

[9] VAN WIJK W. R., BRUIJN P. J., Determination of thermal conductivity and volumetric heat capacity of soils near the surface. Proc. Soil Sci. Soc. (1964) p. 461
[10] Van der Held E. F. M., Van Drunen, F. G., A method of measuring the thermal conductivity of liquids, Physica XV (1949) 865.

[11] HoOper F. C., Lepper F. R., Transient heat flow apparatus for the determination of thermal conductivities, Heating, Piping and Air Conditioning (Aug. 1950) p. 129.

[12] DE VRIES D. A., A nonstationary method for determining thermal conductivity of soil in situ, Soil Sci. 73 (1952) 83.

[13] BUETTNER K., Evaluation of soil heat conductivity with cylindrical test bodies, Trans. Am. Geophys. Union 36 (1955) 831.

[14] Destable P., Etude d'une méthode rapide de mesure des coefficients de conductibilité thermique, Ann. I.T.B.T.P. 11 (1949) 1.

[15] Shannon W. L., Wells W. A., Tests for thermal diffusivity of granular materials, Proc. AM. Soc. Testing and Mater. 47 (1947) 1044.

[16] Mrtchell J. K., KAO T. C., Measurement of soil thermal resistivity. J. of the Geotechnical Engineering Division (Oct. 1978) p. 1307.

[17] Carslaw H. S., Jaeger J. C., Conduction of heat in solids (Oxford Clarendon Press, London) 1959.

[18] Dupain R., Liaison entre les travaux d'aménagement et la géotechnique dans l'estuaire de la Loire : la concavité de Montoir, Thèse de $3^{\mathrm{e}}$ Cycle, Nantes (1982).

[19] KISMI M., DupaIN R., Un sédiment fin de l'estuaire de la Loire : la «jalle», nature et origine sédimentologique. $3^{\text {es }}$ Journées Universitaires de Géotechnique (I.U.T., Saint-Nazaire) 2830/01/1987. 